\title{
Intervención educativa sobre salud bucal en adulto mayor institucionalizado portador de prótesis

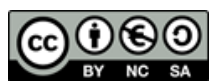

Educative intervention in oral health in institutionalized elderly denture

wearers.

Estela del Rosario Robaina Rivero. ${ }^{1}$, Eliosky Mora Pérez. ${ }^{2}$, Félix Manuel Blanco

Mederos. ${ }^{3}$ \& Domingo Fleitas Echeverría. ${ }^{4}$

Recibido: 10-09-2020 / Revisado: 03-11-2020 /Aceptado: 24-11-2020/ Publicado: 02-01-2021

DOI: https://doi.org/10.33262/anatomiadigital.v4i1.1482

\begin{abstract}
.
Resumen.

The inadecuate wearing of denture leads to El uso inadecuado de las prótesis conlleva a a deterioration of the oral health many un deterioro de la salud bucal muchas veces times seen due to the low level of manifestado por el bajo nivel de knowledge that patients have to carry off conocimientos que tienen los pacientes para good practices of rehabilitation. It was llevar a cabo buenas prácticas de la done a cause experimental study in the elderly home "Celia Sanchez" in the health rehabilitación. Se realizó un estudio cuasi experimental en la Casa de abuelos "Celia area of the Policlinic "7 de December", Jaguey Grande, Matanzas from january to Sánchez Manduley", perteneciente al área de salud del Policlínico "7 de diciembre",

\footnotetext{
${ }^{1}$ Universidad de Ciencias Médicas de Matanzas, Carrera de Estomatología, Matanzas, Cuba. estelarobaina.mtz@nfomed.sld.cu (1) https://orcid.org/0000-0003-0483-964X

2 Universidad de Ciencias Médicas de Matanzas, Carrera de Estomatología, Matanzas, Cuba. ,eliosky.mora@nauta.cu@ https://orcid.org/0000-0002-8276-8456

${ }^{3}$ Universidad de Ciencias Médicas de Matanzas, Carrera de Estomatología, Matanzas, Cuba. felixblanco.mtz@nfomed.sld.cu (Dhttps://orcid.org/0000-0002-0370-9985

${ }^{4}$ Universidad de Ciencias Médicas de Matanzas, Carrera de Enfermería, Matanzas, Cuba .domingofleitas.mtz@nfomed.sld.cu, (Dhttps://orcid.org/0000-0002-3004-9695
} 
december 2019. With gool of applying an educative intervention in oral health, addressed to elderly denture wearers. The populations was formed by 70 elderly, the sample was 63 elderly who fulfilled the inclusion criteria. The variable: sex, schooling level, oral hygiene of the dentures and de level of knowledge about prothetic rehabilitation, before and after the intervention. It was done in stages: diagnostic, intervention and evaluative. The information was picked in a form done for the purpose. To determine the level of knowledge, a questionari was written, proved by experts according to Moriyama criteria. In the study, the females were predominant as well as the primary schooling. The oral hygiene and the hygiene of the dentures were deficient at the beginning, but when finishing the educative intervention, it was classified as efficient in both cases. The educative intervention brought ignorance of old people about aspects of prothetic rehabilitation. The knowledge was risen in all elderly when the intervention ended. It was designed an educative syllabus about oral health focused on institutionalized elderly denture wearers

Keywords: Educative Intervention, Dentures, Elderly
Jagüey Grande, Matanzas, desde enero a diciembre de 2019. Con el objetivo de aplicar una intervención educativa sobre salud bucal, dirigida a adultos mayores portadores de prótesis El universo de estudio estuvo constituido por 70 adultos mayores, la muestra fue de 63 ancianos, que cumplieron los criterios de inclusión. Se analizaron las variables: sexo, nivel de escolaridad, higiene bucal, de las prótesis, y nivel de conocimiento sobre rehabilitación protésica, antes y después de la intervención, esta se ejecutó por etapas: diagnóstica, intervención y evaluativa. $\mathrm{La}$ información se recogió en planilla confeccionada al efecto. Para determinar nivel de conocimientos se elaboró cuestionario, validado por expertos según criterios de Moriyama. En el estudio predominaron las féminas y el grado de escolaridad primaria. La higiene bucal y de las prótesis fue al inicio deficiente, al concluir la intervención educativa se clasificó de eficiente en ambos casos. El diagnóstico educativo arrojo desconocimiento de los ancianos sobre aspectos de la rehabilitación protésica, al finalizar la intervención el conocimiento se elevó en todos los adultos mayores. Se diseñó un programa educativo sobre salud bucal dirigido a adultos mayores institucionalizados portadores de prótesis odontológicas

Palabras claves: Intervención educativa, prótesis odontológica, adulto mayor. 


\section{Introducción.}

Se ha definido el envejecimiento poblacional como el aumento progresivo de la proporción de personas de edad avanzada en una población determinada. (Marques, 2015)

El aumento de personas de edad avanzada constituye la transformación demográfica más importante de la sociedad actual, debido a que los ancianos son el segmento de la población con el crecimiento más rápido del mundo, esto provoca un problema en el campo de la salud y aún más dentro de la Odontología, pues en estas edades, la rehabilitación protésica representa una necesidad de primer orden. (Díaz, Casas y Roldán, 2017)

En Cuba, el cierre del 2018 existía 68068 de 60 años y más, lo que representa el 20,4\% de la población. (Dirección Nacional de Registros Médicos y Estadísticas de Salud, 2019) A pesar de los esfuerzos realizados por los estomatólogos los ancianos siguen aceptando la perdida dentaria como algo inevitable dentro del proceso de envejecimiento por lo que el desdentamiento sigue siendo un problema de salud en el adulto mayor, aumentando la demanda de los tratamientos prótesis rehabilitadores.

El uso inadecuado de las prótesis conlleva a un deterioro de la salud bucal muchas veces manifestado por el bajo nivel de conocimientos que tienen los pacientes para llevar a cabo las buenas prácticas de la rehabilitación. No siempre se puede asumir que el paciente tendrá total comprensión de los beneficios de la prótesis y es improbable que posea el conocimiento necesario para garantizar el cuidado bucal requerido y los procedimientos de mantenimiento, a menos que sea aconsejado adecuadamente. (Díaz et al., 2017)

Con el objetivo de aplicar una intervención educativa sobre salud bucal, dirigida a los adultos mayores portadores de prótesis pertenecientes a la casa de abuelos "Celia Sánchez Manduley", se llevó a cabo la presente investigación

\section{Metodologia.}

Se realizó un estudio cuasi experimental en la Casa de abuelos "Celia Sánchez Manduley”, perteneciente al área de salud del Policlínico "7 de diciembre", Jagüey Grande, Matanzas, desde enero a diciembre de 2019.

El universo de estudio estuvo constituido por 70 adultos mayores, la muestra fue de 63 ancianos, que cumplieron los criterios de inclusión. Se tuvo en cuenta las variables: sexo y nivel de escolaridad, higiene bucal y de las prótesis y nivel de conocimientos sobre rehabilitación protésica antes y después de la intervención. Se realizó un examen inicial a todos los pacientes para llenar un formulario con datos de interés dentro de los que se incluyó la higiene bucal en pacientes dentados y del aparato protésico. 
La higiene bucal se determinó utilizando el Índice de Higiene Bucal de Love, en pacientes parcialmente desdentados. En los desdentados totales y parcialmente desdentados, portadores de prótesis odontológicas se clasificó deficiente., cuando en el momento del examen clínico, se detectaron por lo menos tres factores, como son: lengua saburral, presencia de detritus de alimentos adheridos a la prótesis estomatológica y la mucosa bucal o solamente a la mucosa y la halitosis, de no existir ninguno de ellos, se determinó como eficiente.

La intervención se desarrolló en tres fases: Diagnóstica, de Intervención y Evaluación.

Para el diagnóstico educativo se aplicó a los adultos mayores un cuestionario de ítem test que medió el nivel de conocimientos sobre temas relacionados con la rehabilitación protésica en el adulto mayor, validado por criterio de jueces expertos. Para evaluar la calidad de su construcción y la correspondencia de los ítems con la definición operacional y los factores que la componen, se tuvo en cuenta los criterios de Moriyama.

A partir del diagnóstico inicial se determinaron los objetivos y núcleos temáticos que permitieron el diseño del programa educativo, apoyado en técnicas educativas afectivoparticipativas. Se conformaron grupos pequeños de trabajo para una mejor intervención y que se lograra un mayor impacto en las técnicas utilizadas. El programa contó de 7 módulos de capacitación.

Características del programa educativo: Participativo, cooperativo, educativo, eficaz, diverso y discriminativo. Tuvo como premisa exhortar a los ancianos a la búsqueda de información sobre la rehabilitación protésica odontológica, y promover el intercambio entre los adultos mayores para mejorar la comunicación entre ellos sobre la salud bucal y el uso de la prótesis odontológica.

En la tercera etapa se procedió a constatar los cambios producidos en la higiene bucal y de las prótesis, respectivamente y a la aplicación del cuestionario para evaluar el nivel de conocimientos alcanzado por los adultos mayores después de poner en práctica el programa educativo.

Los datos fueron procesados en una PC Pentium IV con XP y el paquete estadístico SPSS para Windows versión 11.5. Se realizó en la estadística descriptiva distribuciones de frecuencias a todas las variables en estudio, presentando los resultados en frecuencias absolutas y relativas. 


\section{Resultados.}

Tabla 1. Distribución de la muestra según grado de escolaridad y sexo. Casa de Abuelos. Jagüey Grande. 2019.

\begin{tabular}{ccccccc}
\hline \multirow{2}{*}{$\begin{array}{c}\text { Grado de } \\
\text { escolaridad }\end{array}$} & \multicolumn{2}{c}{ Femenino } & \multicolumn{2}{c}{ Masculino } & \multicolumn{2}{c}{ Total } \\
\cline { 2 - 7 } & No & $\%$ & No & $\%$ & No & $\%$ \\
\hline No & - & - & 1 & 3.5 & 1 & 1.6 \\
escolarizado & & 44.1 & 9 & 31 & 24 & 38,1 \\
Primaria & 15 & 29.4 & 8 & 27.5 & 18 & 28,6 \\
Secundaria & 10 & 8,8 & 3 & 10.4 & 6 & 9.5 \\
Preuniversitario & 3 & 8,8 & 6 & 20,7 & 9 & 14.3 \\
Técnico medio & 3 & 8,8 & 2 & 6.9 & 5 & 7.9 \\
Universitario & 3 & $\mathbf{5 4}$ & $\mathbf{2 9}$ & $\mathbf{4 6}$ & $\mathbf{6 3}$ & $\mathbf{1 0 0}$ \\
Total & $\mathbf{3 4}$ & & & & & \\
\hline
\end{tabular}

El grado de escolaridad se refleja en la Tabla 1, en ambos sexos el mayor porciento de sujetos tenía escolaridad primaria, lo que constituyó 38,1 \% de la muestra. El sexo femenino sobre el sobre el masculino con un $54 \%$.

El grado de escolaridad es una variable a tener en cuenta cuando se realiza una intervención educativa, mientras más elevado sea el grado de escolaridad mejor se apropiarán de los conocimientos, mejor captan los mensajes educativos que se les transmite y es más factible la intervención con un mayor impacto en los resultados alcanzados.

Esmeriz, Meneghim y Ambrosano, (2012) demuestran relación entre la autopercepción de salud bucal y el nivel de escolaridad de los individuos, observando una autopercepción buena en los individuos de mayor nivel de escolaridad, aunque no en todos los casos los individuos de mayor nivel de escolaridad, de manera individual, presentaron mejor estado de salud bucal.

Los resultados coinciden con Alemar, González, Gross, Martínez, Pol y Marti, (2020) ellos describen que el nivel de escolaridad que prevalece en su investigación en adultos mayores fue de primaria., seguidos de la secundaria. 
Tabla 2(a). Higiene bucal antes y después

\begin{tabular}{ccccc}
\hline & \multicolumn{3}{c}{ Higiene bucal } \\
\cline { 2 - 5 } Clasificación & \multicolumn{2}{c}{ Antes } & \multicolumn{2}{c}{ Después } \\
\cline { 2 - 5 } & No & $\%$ & No & $\%$ \\
\hline Eficiente & 28 & 44.4 & 61 & 96.8 \\
Deficiente & 35 & 55.6 & 2 & 3.2 \\
Total & $\mathbf{6 3}$ & $\mathbf{1 0 0}$ & $\mathbf{6 3}$ & $\mathbf{1 0 0}$ \\
\hline
\end{tabular}

Tabla 2 (b). Higiene de las prótesis odontológicas antes y después.

\begin{tabular}{lcccc}
\hline \multirow{2}{*}{ Clasificación Higiene de las prótesis } \\
\cline { 2 - 5 } & \multicolumn{3}{c}{ Antes } & \multicolumn{2}{c}{ Después } \\
\cline { 2 - 5 } & No & $\%$ & No & $\%$ \\
\hline Eficiente & 21 & 33,3 & 58 & 92.1 \\
Deficiente & 42 & 66,7 & 5 & 7.9 \\
Total & $\mathbf{6 3}$ & $\mathbf{1 0 0}$ & $\mathbf{6 3}$ & $\mathbf{1 0 0}$ \\
\hline
\end{tabular}

En las tablas 2(a) y 2(b) se ilustra la distribución de la muestra de acuerdo a la higiene bucal y de las prótesis. Antes de la intervención predominó la higiene bucal deficiente en el 55.6\% de la muestra, después sólo el 3,2\% de los ancianos continúo con deficiente higiene bucal. Por su parte, antes de la intervención prevaleció la higiene deficiente de las protesis en el $66.7 \%$ de la muestra, después este parámetro se clasificó de eficiente en el $92.1 \%$, de la población en estudio.

Los resultados son similares a los de (Naranjo, Céspedes, Sablón, Hechavarría y Castronuño, 2019), que realizaron una intervención educativa dirigida a la promoción de salud bucal en el adulto mayor, en Banes, Holguín, ellos sostienen que el nivel de conocimientos que refieren los encuestados sobre higiene bucal, en un momento inicial solo el $27,6 \%$ tenían un nivel adecuado de conocimientos, mientras que, al final ya se alcanza el $93,1 \%$ de buena higiene bucal. Se refleja, además, las prácticas de cuidado e higiene de la prótesis dental de los 15 adultos portadores de prótesis (51,7\%); antes de la intervención solo 6 (40\%) tenían dominio de cómo mantener su cuidado e higiene, después de la intervención educativa, la totalidad de los portadores (100\%) cuidaban de forma correcta sus prótesis.

Con respecto a la higiene bucal de los adultos mayores, antes de la intervención, se encuentra el mayor porcentaje en la categoría de regular, 67,3\%, y después de la intervención, predomina la categoría de buena, 91,8 \%, referente a las prótesis antes de la intervención es regular en el 67 \% y después de la intervención se clasifica de buena en el 
$92 \%$ de los pacientes según Cobos, Gutiérrez, Paterson y Zamora (2015). Demostrándose semejanza en la presente investigación.

También se concuerda con Moya, Chappuzeau, Caro y Monsalves (2012) quienes encuentran un $43 \%$ de adultos mayores con higiene oral regular y un $23,8 \%$ deficiente, al estudiar la "Situación de salud oral y calidad de vida de los adultos mayores".

Charry, Gómez y Roa, (2015), al aplicar el índice de Higiene bucal a 19 adultos mayores reportan una higiene bucal deficiente, con un porcentaje del 84.2\%. En la fase de medición de resultados posterior a la aplicación de la estrategia educativa, en esta población se observa una mejoría en las condiciones de higiene bucal en un $68 \%$.

Uno de los temas sobre los que más se insiste en el diario bregar de la educación para la salud, es el eficiente control de la higiene bucal, así como de la higiene de las prótesis. No sólo las actividades educativas deben dirigirse al adulto mayor, que puede tener limitaciones físicas y/o mentales propias del de cursar del tiempo, es preciso involucrar en el proceso educativo a los familiares y los cuidadores. En el caso de los ancianos institucionalizados los trabajadores de la institución deben ser parte activa del proceso de capacitación y los administrativos deben garantizar las condiciones para que los adultos mayores realicen una correcta higienización de la cavidad bucal y de las prótesis.

Tabla 3. Conocimientos antes y después de la intervención.

$$
\mathbf{n}=63
$$

\begin{tabular}{|c|c|c|c|c|c|c|c|c|c|c|c|c|}
\hline \multirow{3}{*}{ Conocimiento } & \multicolumn{8}{|c|}{ Antes } & \multicolumn{4}{|c|}{ Después } \\
\hline & \multicolumn{2}{|c|}{ Conoce } & \multicolumn{2}{|c|}{$\begin{array}{c}\text { No } \\
\text { conoce }\end{array}$} & \multicolumn{2}{|c|}{ Total } & \multicolumn{2}{|c|}{ Conoce } & \multicolumn{2}{|c|}{$\begin{array}{c}\text { No } \\
\text { conoce }\end{array}$} & \multicolumn{2}{|c|}{ Total } \\
\hline & No & $\%$ & No & $\%$ & No & $\%$ & No & $\%$ & No & $\%$ & No & $\%$ \\
\hline Sobre higiene bucal y de las prótesis & 4 & 6,3 & 59 & 93,7 & 63 & 100 & 62 & 98,4 & 1 & 1,6 & 63 & 100 \\
\hline Sobre uso y cuidado de las prótesis. & 11 & 17,5 & 52 & 82,5 & 63 & 100 & 63 & 100 & 0 & - & 63 & 100 \\
\hline $\begin{array}{l}\text { Sobre lesiones asociadas al uso de } \\
\text { las prótesis. }\end{array}$ & 1 & 1,6 & 62 & 98,4 & 63 & 100 & 60 & 95,2 & 3 & 4,8 & 63 & 100 \\
\hline $\begin{array}{l}\text { Sobre frecuencia de visitas al } \\
\text { odontólogo }\end{array}$ & 0 & - & 63 & 100 & 63 & 100 & 63 & 100 & 0 & - & 63 & 100 \\
\hline
\end{tabular}

Al observar los resultados de la tabla 3 se muestra que antes de la intervención educativa el $100 \%$ de los adultos mayores no tenían conocimientos sobre la frecuencia de visitas al estomatólogo. En orden ascendente continua el conocimiento sobre lesiones asociadas al 
empleo de las prótesis, alcanzado por el 1,6\%, el conocimiento sobre higiene de las prótesis odontológicas sólo lo tenía el 6,3 \% de los adultos mayores. La mayor representatividad de conocimiento inicial fue sobre el uso y cuidado de las prótesis estomatológicas, aunque solo fue demostrado por el 17,5 \% de ellos. Después de la intervención se elevo el nivel de conocimientos en todos los aspectos, resalto el $100 \%$ alcanzado respecto al uso y cuidado de las prótesis y frecuencia de visitas al odontólogo.

Sobre la higiene bucal, uso y mantenimiento de las prótesis, antes de la intervención educativa prevalecen los conocimientos inadecuados en 23 de los 30 adultos mayores (76.7\%), o sea 5 de los adultos mayores; sin embargo, después de la intervención se logra un $91.3 \%$ que unido a los 7 adultos mayores que tienen y mantienen los conocimientos adecuados se eleva a un $93.3 \%$ por lo cual los cambios fueron significativos. De esta forma solamente 2 adultos mayores al culminar la intervención continuaron con conocimientos inadecuados representando al 6.7\%. La pregunta de mayor desconocimiento sobre el tema fue la relacionada con la frecuencia y el método utilizado para la higiene de las prótesis. (Alemar et al., 2020). Existe concordancia del estudio con los resultados anteriores.

Además, refiere (Alemar et al., 2020) que, al inicio de la intervención sobre las patologías más frecuentes en la cavidad bucal, 93,3\% de los ancianos tienen conocimientos inadecuados, pero después de la intervención estos se tornan adecuados en un 96,7\%, los cambios de conocimientos obtenidos resultaron altamente significativo estadísticamente $(\mathrm{p}<0,01)$.

Al finalizar una intervención educativa en adulto mayor se logra que el 93,1\% alcanzara un nivel de conocimiento de salud e higiene bucal adecuada. La totalidad de los que tienen prótesis dental cuidan las mismas de forma correcta y con relación al conocimiento sobre lesiones bucales un 86,2\% alcanzó un adecuado nivel, según (Naranjo et al., 2019) Existe correspondencia con los resultados obtenidos.

También son similars a Bermúdez, Martínez e Ibáñez, (2015), quienes evidencian que los pacientes en estudio fueron calificados de mal en cuanto a conocimiento sobre higiene bucal y de las prótesis $(90,6 \%)$.

Al referirse a las visitas al odontólogo un pequeño por ciento, respondió que nunca iban al odontólogo porque eran portadores de prótesis; (Herrera, 2018), esto es incorrecto lo hacen por desconocimiento, pues, aunque la persona no tenga dientes naturales debe acudir a consulta para revisión de las estructuras que están en contacto con la prótesis, en busca de lesiones que pudieran ser la antesala del cáncer bucal, siendo esta otras de las medidas preventivas en Odontología. Es una obligación y deber, por parte de los profesionales de la salud, de educar a la población sobre temas que todavía desconocen, para de esta manera erradicar las actitudes negativas y prejuiciosas que existen aún en la población adulta mayor. Se refleja analogía de los resultados obtenidos en la investigación actual. 
Gonzabay, (2016). Refiere en cuanto a las instrucciones por parte del odontólogo, después de la rehabilitación protésica resultan ser bastante necesarias, ya que el $62 \%$ nunca ha recibido ningún tipo de orientación al respecto. En otro estudio sobre evaluación de los hábitos de higiene y actitudes en pacientes portadores de prótesis, un $49 \%$ de los pacientes indica que no habían sido informados por su odontólogo. (Cakan, Yuzbasioglu, Kurt, Turunc, Akbukt y Aydin, 2015).

De manera general todos los investigadores coinciden en que existe desconocimiento de los adultos mayores sobre temas vitales relacionadas con la salud bucal, por eso, al realizar intervenciones educativas se corrobora el desconocimiento inicial, al concluir las mismas siempre se eleva hasta alcanzar niveles adecuados, se ratifica también lo referido por Cobos et al.,(2015) que en relación con el nivel de conocimiento de los ancianos de las casas de abuelos de Mayarí, la mayoría de ellos antes de la intervención, (61,2\%), se ubican en la categoría de regular, después de la intervención, el 95,9 \% alcanza la categoría de bien.

La población necesita conocer más sobre aspectos relacionados con la higiene bucal, en primer lugar, la importancia que tiene el cepillado dental como medida preventiva y eficaz, no solo para evitar enfermedades sino para que el individuo disfrute del placer de una boca limpia, de una higiene bucal correcta; lo que producirá un doble efecto: bienestar y prevención de las enfermedades más frecuentes de la cavidad bucal, evitando la pérdida dentaria que tanto afecta las diversas funciones del aparato estomatognático, entre ellas la estética.

Los problemas de salud bucal en los adultos mayores deben tomarse en cuenta, pues en esta etapa de la vida cobran mayor importancia debido al impacto que tienen en la calidad de vida, así como en el mayor riesgo de tener consecuencias más severas y complejas que requieren de atención especializada. Es por ello que se hace necesario el diseño e implementación de programas de intervención educativa dirigidos a este grupo de la población.

El Odontólogo General Integral, y el especialista de Prótesis debe brindar toda la información que necesita la población respecto a su salud bucal; si bien es cierto, que el individuo es el máximo responsable de su salud, ellos, deben brindar el conocimiento y las herramientas para fomentar y mantener un adecuado estado de salud bucal y de las prótesis.

\section{Conclusiones.}

- En el estudio predominaron las féminas y el grado de escolaridad primaria. 
- La higiene bucal y de las prótesis fue al inicio deficiente, al concluir la intervención educativa se clasificó de eficiente en ambos casos.

- El diagnóstico educativo arrojó desconocimiento de los adultos mayores sobre aspectos de la rehabilitación protésica, al finalizar el programa educativo el conocimiento se elevó en todos los adultos mayores.

- Se diseñó un programa educativo sobre salud bucal dirigido a adultos mayores institucionalizados portadores de prótesis odontológicas

\section{Referencias bibliográficas.}

Alemar, YC., González, T., Gross, Y., Martínez, MN., Pol L. \& Marti, M. (2020) Instructivo Educativo "Sonríele a la edad" en pacientes adultos mayores. Recuperado de https://www.revista-portalesmedicos.com/revista-medica/sonriele-ala-edad-adultos-mayores/7/.

Bermúdez, R., Martínez, G., Ibáñez I. (2015). Evaluación de conocimientos sobre estomatitis subprotésica en pacientes y profesionales de la Clínica Docente de Especialidades. Revista Medicentro Electrónica., 19(4)

Cakan, U., Yuzbasioglu, E., Kurt,H., Turunc, R., Akbukt, A. \& Aydin K.(2015). Assessment of hygiene habits ands attitudes among removible parcial dentare Sears in a university hospital. Nigerian Journal of Clinical Practice, 511-515.

Charry, AP. Gómez, A., Roa, S. (2015). Diseño e implementación de una estrategia educativa en salud bucal para las personas mayores de tres hogares apoyados por la alcaldia de Villavicencio. Tesis para optar por el título de Odontólogo Universidad Cooperativa Odontológica. Colombia.

Cobos, I., Gutiérrez, M., Paterson, E. \& Zamora, N. (2015) Intervención educativa sobre estomatitis subprótesis en el adulto mayor. Holguín., (19)3.Recuperado de http://scielo.sld.cu/scielo.php?script=sci_arttext\&pid=S1560-43812015000300005.

Diaz, A., Hussain, A., Marcos, A., \& Roque, A. (2011). A biotechnological perspective on the application of iron oxide magnetic colloids modified with polysaccharides., Biotechnology Advances 29 (29), 142-155.

Díaz, CV., Casas, I., Roldán. J. (2017) Calidad de vida relacionada con salud oral: Impacto de diversas situaciones clínicas odontológicas y factores socio-demográficos. Revisión de la literatura. Int. J. Odontostomat., 11(1) ,31-39.

Dirección Nacional de Registros Médicos y Estadísticas de Salud, (2019). Anuario Estadístico de salud 2018. 17-21 47ed. La Habana: CEDISAP. UEB Producciones gráficas.

Esmeriz, CE, Meneghim, MC, Ambrosano, GM.(2012). Self perception of oral health in non institutionalised elderly of Piracicaba city, Brazil. Gerodontology., 29(4),281-9.

Gonzabay, D. (2016). Conocimiento de los pacientes sobre el cuidado de prótesis fija/removible y creación de manual destinado a la promoción de higiene y cuidado 
protésico. Trabajo de titulación para optar por el Título de Odontóloga. Universidad de las Américas. Quito. Ecuador.

Herrera, IB. (2018). Salud bucal y funcionamiento familiar en personas del municipio Plaza de la Revolución de La Habana, Cuba. Universidad Odontológica de La Habana. Cuba., 38(79). Recuperado de https://doi.org/10.11144/Javeriana.uo3779.sbff

Marques, J. (2015). Actualizar criterios sobre envejecimiento tiene una significación especial. Recuperado de http://www.sld.cu/entrevista/2015/04/14/actualizarcriterios-sobre-envejecimiento-tiene-una-significacion-especial.

Moya, P., Chappuzeau, E., Caro, JC. \& Monsalves, MJ. (2012). Situación de salud oral y calidad de vida de los adultos mayores. Situación de salud oral y calidad de vida de los adultos mayores. Revista Estomatológica Herediana., 22(4):197-202.

Naranjo, HA., Céspedes, JL. Sablón, LM. , Hechavarría, MR. \& Castronuño, L. (2019). Intervención educativa dirigida a la promoción de salud bucal en el adulto mayor. Revista 16 de abril., 58 (274), 98- 104 


\section{PARA CITAR EL ARTÍCULO INDEXADO.}

Mora Pérez, E., Robaina Rivero, E. del R., Blanco Mederos, F. M., \& Fleitas Echeverría, D. (2021). Intervención educativa sobre salud bucal en adulto mayor institucionalizado portador de prótesis. Anatomía Digital, 4(1), 65-76. https://doi.org/10.33262/anatomiadigital.v4i1.1482

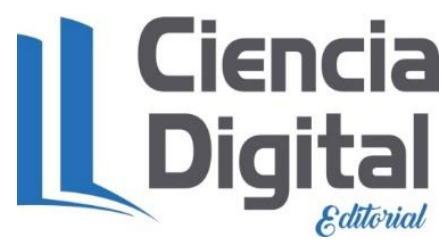

El artículo que se publica es de exclusiva responsabilidad de los autores y no necesariamente reflejan el pensamiento de la Revista Anatomía Digital.

El artículo queda en propiedad de la revista y, por tanto, su publicación parcial y/o total en otro medio tiene que ser autorizado por el director de la Revista Anatomía Digital.
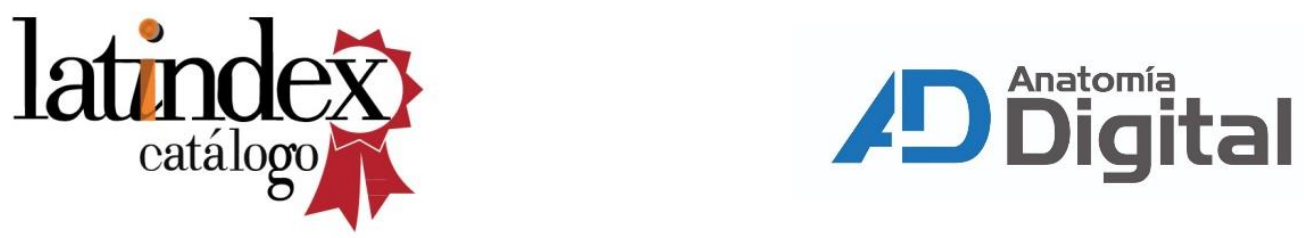